\title{
Retraction Note to: Effect of coupling asynchronous acoustoelectric effects on the corrosion behavior, microhardness and biocompatibility of biomedical titanium alloy strips
}

\author{
Xiaoxin Ye $\mathrm{e}^{1,2} \cdot$ Guoyi Tang ${ }^{1,2}$
}

Published online: 5 October 2016

(C) Springer Science+Business Media New York 2016

\section{Retraction Note to: J Mater Sci: Mater Med (2015) 26:53 \\ DOI 10.1007/s10856-014-5371-5}

The article entitled "Effect of coupling asynchronous acoustoelectric effects on the corrosion behavior, microhardness and biocompatibility of biomedical titanium alloy strips", published in Journal of Materials Science: Materials in Medicine, Volume 26: 53 (DOI 10.1007/s10856-0145371-5), is hereby retracted at the request of the Editor-inChief and with the agreement of the authors.

Following a complaint about the republishing of unreferenced data in several journals including Journal of Materials Science: Materials in Medicine, the following issues were detected: there are textual similarities and duplication of images in the above-mentioned paper and in "The effect of electropulsing induced gradient topographic oxide coating of Ti-Al-V alloy strips on the fibroblast adhesion and growth", authored by Xiaoxin Ye, Zion Tse,
Guoyi Tang and Guolin Song, and published in Surface and Coatings Technology, notably Figures 2 and 3(a) in the latter article appear as Figures 5 and 7 in the Journal of Materials Science: Materials in Medicine paper without reference to the original publication.

In addition, similarities in other figures (for example, Figures 8 and 9) have been noted with a number of other publications. For example:

Effect of electroplastic rolling on deformability, mechanical property and microstructure evolution of Ti6Al-4V alloy strip, Xiaoxin Ye, Zion T.H. Tse, Guoyi Tanga, Guolin Song, Materials Characterization, Volume 98, December 2014, Pages 147-161.

Effect of electropulsing treatment and ultrasonic striking treatment on the mechanical properties and microstructure of biomedical ti-6Al-4V alloy, Xiaoxin Ye, Yongda Ye, Guoyi Tang, Journal of the Mechanical Behavior of Biomedical Materials, Volume 40, December 2014, Pages 287-296.

The online version of the original article can be found under doi:10.1007/s10856-014-5371-5.

Guoyi Tang

tanggy@mail.sz.tsinghua.edu.cn

Xiaoxin Ye

xiaoxinye905@gmail.com

1 Advanced Materials Institute, Graduate School at Shenzhen, Tsinghua University, Shenzhen 518055, People's Republic of China

2 Key Laboratory for Advanced Materials of Ministry of Education, Department of Materials Science and Engineering, Tsinghua University, Beijing 100084, People's Republic of China 